\title{
IMAGING OF RECONNECTION PROCESSES IN HARD X-RAYS
}

\author{
Zdenek Švestka* and Giannina Poletto** \\ ${ }^{*}$ Laboratory for Space Research, Beneluxlaan 21, 3527 HS \\ Utrecht, The Netherlands \\ ** Osservatorio Astrofisico di Arcetri, Largo Enrico Fermi 5, \\ 50125 Firenze, Italy
}

\author{
ABSTRACT
}

The Hard X-ray spectrometer aboard the SMM detected several events of energy release late in the development of two-ribbon flares. One such event, at 21:12 UT on 21 May, 1980 ( 20 min after the flare onset and $15 \mathrm{~min}$ after the peak of the impulsive phase) is studied in detail. The site of new brightening first became visible in hard X-rays $(>22 \mathrm{keV})$ and only afterwards showed up at lower energies. It was clearly located high in the corona so that one can identify it with energy release at the tops of newly formed post-flare loops. Thus, if the Kopp and Pneuman model of the loop formation is adopted, we may have imaged here a reconnection process in the solar corona. An attempt is made to estimate physical parameters at the reconnection site.

\section{GROWTH OF POST-FLARE LOOPS}

X-ray images of several two-ribbon flares obtained by the Hard $x-r a y$ Imaging Spectrometer (HXIS) aboard the SMM have revealed that the growth of post-flare loops is not a continuous process. Instead, the tops of loops stay at a given altitude for quite a while and rather suddenly a set of new loops appears at higher altitude. A detailed analysis indicates that progressively higher loops may be sequentially formed all the time, but there are bunches of loops that appear (i.e. probably reconnect) at about the same time, and these then dominate the brightness distribution in X-rays.

Examples of this behavior are shown in Figure 1, for the wel1-known flare of 21 May, 1980 (position $15 \mathrm{~W}, 14 \mathrm{~s}$ ). The flare started at 20:50 UT /1/ and the X-ray emission originated southward of the $\mathrm{H}_{\|}=0$ line at a distance $d \simeq 5000 \mathrm{~km}$; this, taking into account projection effects, would correspond for radial loops to an altitude of $15000 \mathrm{~km}$ above the $\mathrm{H}_{\|}=0$ line. At 20:55, simultaneously with a very steep rise in $x$-ray flux, the maximum brightness shifted to $d \simeq 9000 \mathrm{~km}$, and stayed at this altitude until 21:12 UT, when a second rise heaved the loop tops to $\mathrm{d} \simeq 15000 \mathrm{~km}$, $i$.e. radial altitude of $43000 \mathrm{~km}$. Temperature in the flare peaked at 20:58 UT and hard X-rays had three maxima (with decreasing flux) at 20:56, 20:58, and 21:01 UT. The hard $x$-ray burst practically decayed at 21:05 UT /1,2/. Thus the second rise of the loop tops at 21:12 UT occured several minutes after the end of the impulsive phase, which characterizes the main release of energy in the flare, and some $15 \mathrm{minutes}$ after the maximum of the energy release. We will now analyse this second jump in altitude in more detail.

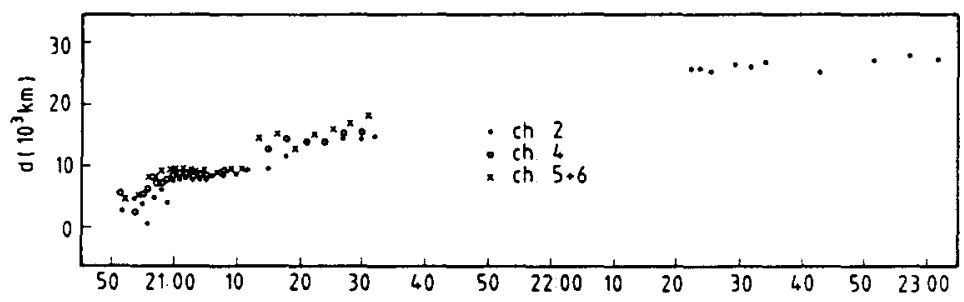

Fig. 1. Time variation of the distance $d$ of the brightest part of the flare of 21 May 1980 (tops of the $\mathrm{X}$-ray loops) from the $\mathrm{H}_{\|}=0$ line, in projection on the solar disc. Different symbols refer to observations in HXIs channels 2 (5.5-8.0 keV), 4 ( $11.5-16.0$ $\mathrm{keV})$ and $5+6(16-30 \mathrm{keV})$. There is a data gap between $21: 33$ and $22: 20$ UT. 


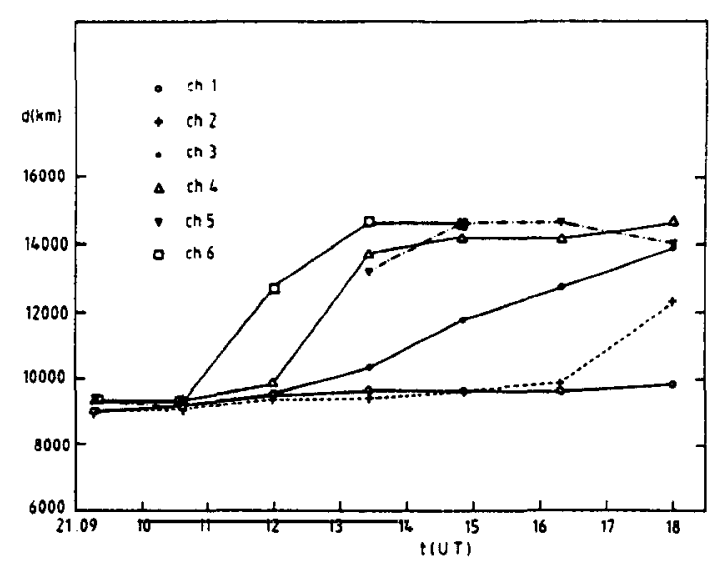

Fig. 2. A detail of the variations of d during the second rise in figure 1 , in different energy channels of HXIS: $1(3.5-5.5 \mathrm{keV}), 2,3(8.0-11.5 \mathrm{keV}), 4,5(16-22$ $\mathrm{keV})$, and $6(22-30 \mathrm{keV})$.

\section{RECONNECTION PROCESS HIGH IN THE CORONA}

Figure 2 shows a detail of the second rise, in various energy bands. One can see clearly that the high-energy bands showed the rise much earlier while the low-energy bands followed only later. Since the sensitivity to temperature variations increases with energy, the measurements reveal sudden heating at higher altitudes, i.e. a new energy release high in the corona in the late post-maximum phase of the flare.

The sequence of HXIS images of the flare in Figure 3 shows what actually happened. The image in band 4 at 21:09:17 UT represents the typical situation seen in all the bands before: a maximum of brightness at a distance $d \simeq 9000 \mathrm{~km}$ from the $H_{\|}=0$ line. In band 6 , at that time, one can see already an extension of the flux-contours to the south. This extension is more pronounced at 21:10:38, and finally a new maximum is formed, more to the sw, i.e. higher in the corona, at 21:12 UT (marked by letter A). This is the site we begin to see at that time at $d \simeq 13000-15000 \mathrm{~km}$ in Figures 1 and 2. At lower energies this new brightening occurs successively later, as we indicate by arrows.

The newly formed $x-r a y$ emission (A) came predominantly from two pixels of HxIs fine field of view, Nos. 367 and 383. We have recorded the $x$-ray flux from these two pixels only, from 21:02 UT through 21:30 UT, and computed the ratios of fluxes in neighboring HxIs energy bands: $6 / 5,5 / 4, \ldots ., 2 / 1$. Using the new HXIS calibration /3/ we computed temperatures from these ratios and plotted them as functions of time in Figure 4 . We had to use $3-\mathrm{min}$ means smoothed by $1-\mathrm{min}$ steps, in order to get statistically significant counts in band 6 . Therefore, any short time variations, if present, have been smoothed out.

The temperature curves in Figure 4 show the same effect as the images in Figure 3 : there is an enhancement in pixels 367 and 383 which peaks at $21: 13$ UT for the ratio $6 / 5$ and progressively later $z$ tower onersiea.

The only explanation we can offer for this behaviour is that first a small source of very high temperature formed in the corona. As an illustration, let us enhance the temperature of a small volume in the corona from $20 \times 10^{6}$ to $50 \times 10^{6} \mathrm{~K}$. Then the $\mathrm{x}$-ray flux from this volume rises by a factor 2230 in tXIS band 6, a factor 275 in band 5, 60 in band 4 , but only by a factor 3 in band $1 / 3 /$. Therefore, we can see the source in band 6 in spite of its small dimensions, whereas in lower energy bands we must wait till its emission measure increases, i.e. till more electrons take part in the heating process. This clearly indicates that we have imaged here a process of energy release accomplished in a small volume of plasma. An obvious candidate is reconnection of field lines, in apparent agreement with the Kopp and Pneuman model of the formation of post-flare loops $/ 4 /$.

\section{ANALYSIS OF THE NEW SOURCE OF ENERGY RELEASE}

The new source of emission is projected on an $x-r a y$ background in the pixels 367 and 383 , which is very strong in band 1 and progressively weaker in the higher energy bands. Even band 6 shows a background flux which is mainly due to the source in the neighboring pixel located nearer to the $H_{\|}=0$ line (as the triangle response of the HXIs collimator has FWHM $=8 "$, but base $=16^{\prime \prime}$ ). However, another part is apparently real, indicating that some weaker loops had already penetrated with their tops into the pixels 367 and 383 earlier. Thus the 


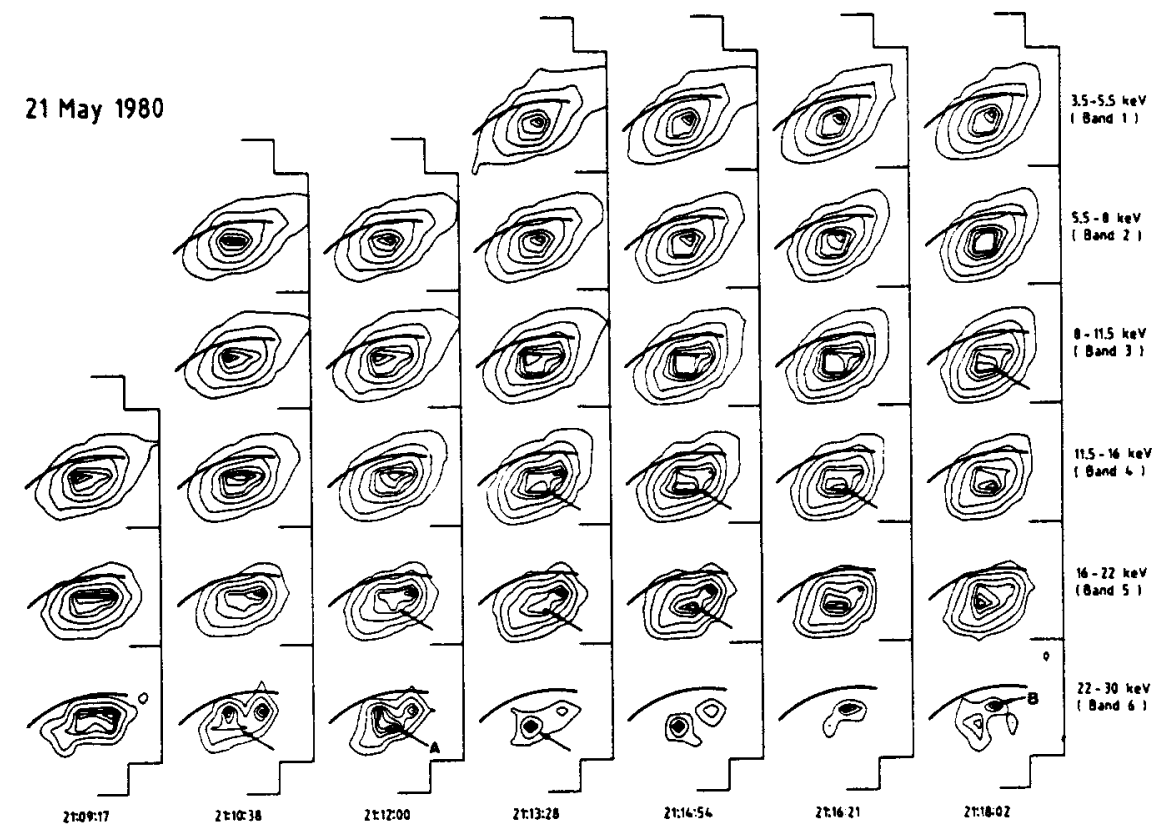

Fig. 3. Images of the flare of 21 May 1980 in HxIs bands 1 - 6 during the period plotted in Figure 2. The feature $A$ is a new maximum of brightness formed at $21: 12$ UT in band 6 and later on in the lower bands. Feature $B$, on the other hand, is an artifact that must be ignored in all images where it appears. (It is a pixel with abnormally high gain at higher energies). The $H_{\|}=0$ line is marked in all the images. North is up, west is to the right.

temperatures in Figure 4 are underestimated, but we may still use them to draw some conclusions about the cooling process: even with the underestimated temperature values the observed cooling time is too slow to be interpreted as the cooling of a single loop - one needs $\mathrm{n} \geq 2 \times 10^{12} \mathrm{~cm}^{-3}$ for purely conductive cooling, and, of course, for these densities still faster radiative cooling would prevail. Therefore, the process we imaged had to consist of a series (bunch) of loops which were sequentially excited and sequentially cooled, thus making the observed cooling time fictitiously longer.

For any further analysis we will use the $6 / 5$ curve of Figure 4 , which is repeated in Figure 5, with 1-sigma error bars added. Below it we show the time variation of the emission measure $Y$ determined from counts in channels 6 and 5 . The emission measure was decreasing up to the time of maximum temperature and began to increase after that. The decrease can be understood if the emission consisted of two components: an extensive cooler background and a small new hot source of energy release. As temperature in the new source increases, a progressively larger fraction of radiation comes from the hot source, of which the emission measure is small. After the maximum is reached, the source expands, more electrons become involved (also through evaporation perhaps) so that the emission measure increases.

There are four unknown parameters which enter into the process: temperature (Tb) and emission measure $\left(Y_{b}\right)$ of the background radiation, and temperature ( $T_{s}$ ) and emission measure $\left(Y_{s}=m Y_{b}\right.$ with $\left.m<<1\right)$ of the new source. Since we do not have enough conditions to determine uniquely all four unknown parameters, we just restrict ourselves to an illustrative example: let us suppose that $T_{b}=25 \times 10^{6} \mathrm{~K}$ and $T_{s}=50 \times 10^{6} \mathrm{~K}$ at 21:13:19 UT, when $T$ is maximum and $Y$ minimum in Figure 5. Then $m=0.0073, Y_{b}=4.1 \times 10^{47} \mathrm{~cm}^{-3}$, and $Y_{s}=3.0 \times 10^{45} \mathrm{~cm}^{-3}$. This emission measure of the source yields the combinations of volume and density given in Table 1. A more detailed modelling of Figure 5 is in progress.

TABLE 1 Size of the new source for various densities

$\begin{array}{llll}\text { Density }\left(\mathrm{cm}^{-3}\right) & 10^{9} & 10^{10} & 10^{11} \\ \text { Volume }\left(\mathrm{cm}^{-3}\right) & 3.0 \times 10^{27} & 3.0 \times 10^{25} & 3.0 \times 10^{23} \\ \text { Radius of the } & & & \\ \text { volume if } & 9000 & 1900 & 415\end{array}$




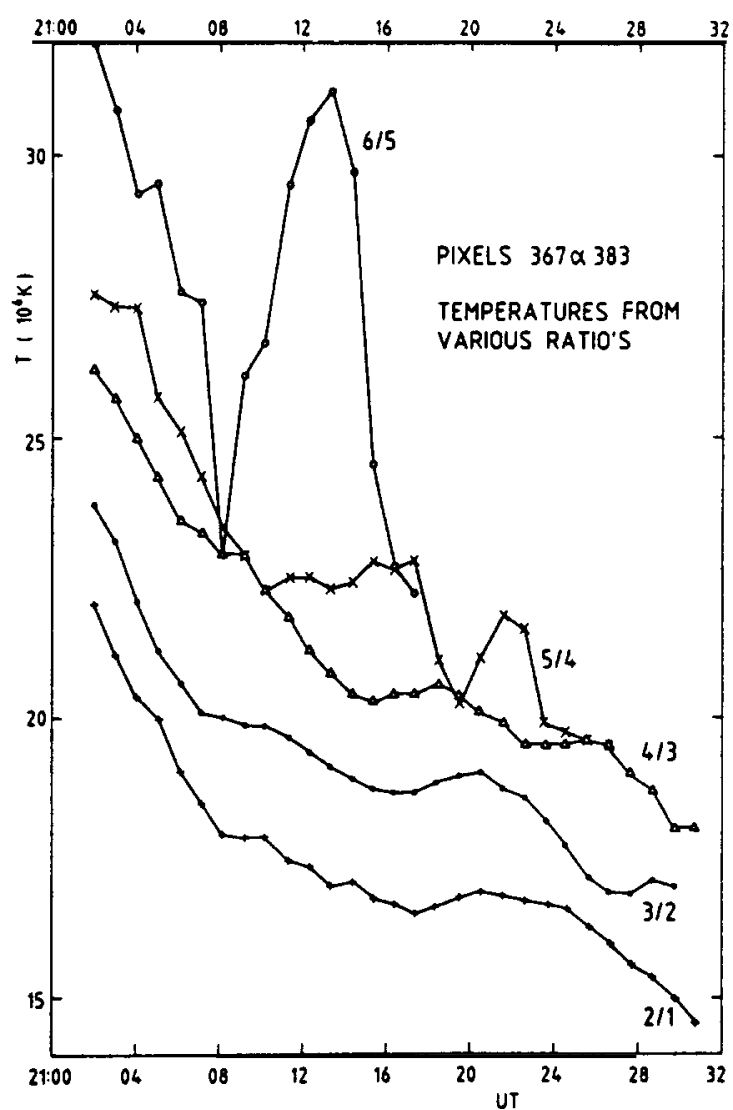

Fig. 4 .

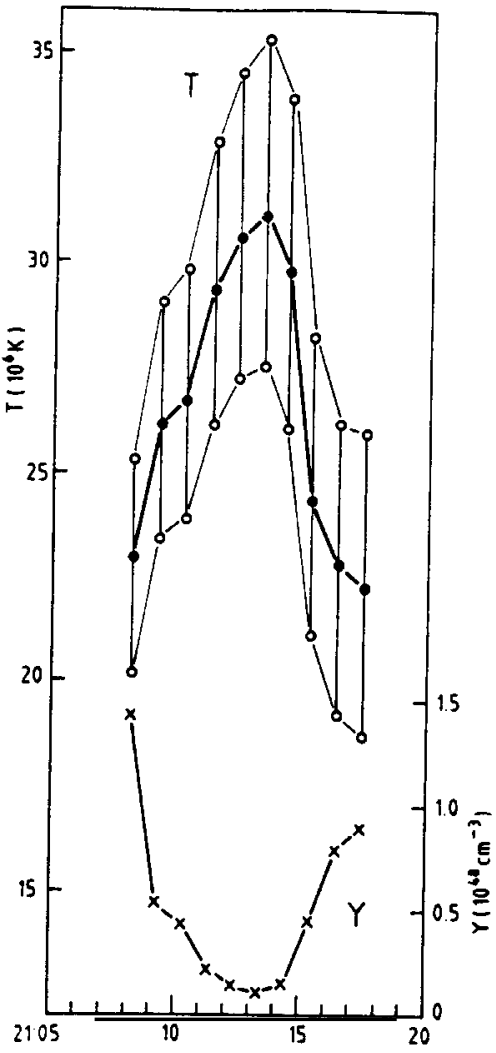

Fig. 5 .

Fig. 4. Temperature deduced from the ratios of different HxIs energy bands in pixels 367 and 383 which were the site of the new X-ray brightening at 21:12 UT. 6/5, e.g., means the ratio of counts in band 6 over band 5 .

Fig. 5. Variations of temperature ( $T$ ) and emission measure ( $Y$ ) in HXIS pixels 367 and 383, determined from the ratio of counts in bands 6 and $5(22-30 \mathrm{kev} / 16-22 \mathrm{keV})$ from 21:08 to 21:18 UT. The curve for $T$ is taken from Figure 4, and 1-sigma error bars are added.

\section{ACKNOWLEDGEMENT}

The development and construction of HXIS /5/ was made possible by support from the Netherlands Ministry for Education and Science, and the Science and Engineering Research Council of the United Kingdom.

\section{REFERENCES}

1. P. Hoyng, A. Duijveman, M.E. Machado, D.M. Rust, Z. Svestka, A. Boelee, C. de Jager, K.J. Frost, H. Lafleur, G.M. Simnet,, H.F. van Beek, and B.E. Woodgate, Origin and location of the Hard X-Ray Emission in a Two-Ribbon Flare, Astrophys. J. 246, L155 (1981)

2. A. Duijveman, P. Hoyng, and M.E. Machado, X-ray Imaging of Three Flares during the Impulsive Phase, Solar Phys. 81, 137 (1982)

3. R. Mewe, E.H.B.M. Gronenschild, and G.H.J. van den Dort, Calculated X-Radiation from Optically Thin Plasmas, V. Improved Atomic Data, Astron. Astrophys. Suppl., submitted.

4. R.A. Kopp and G.W. Pneuman, Magnetic Reconnection in the Corona and the Loop Prominence Phenomenon, Solar Phys. 50, 85 (1976)

5. H.F. van Beek, P. Hoyng, H. Lafleur, and G. Simnet,, The Hard X-Ray Imaging Spectrometer (HXIS), Solar Phys. 65,39 (1980) 